\title{
Immunogenicity of a Fap2 peptide mimotope of Fusobacterium nucleatum and its potential use in the diagnosis of colorectal cancer
}

\author{
Leonardo A. Guevarra Jr ${ }^{1,2^{*}}$, Andrea Claudine F. Afable ${ }^{1}$, Patricia Joyce O. Belza ${ }^{1}$, Karen Joy S. Dy ${ }^{1}$,
} Scott Justin Q. Lee', Teresa T. Sy-Ortin ${ }^{3}$ and Pia Marie S. P. Albano ${ }^{2,4}$

\begin{abstract}
Background: The role of Fusobacterium nucleatum Fap2 protein in the development of colorectal cancer has recently been explained. Fap2, when bound to the human inhibitory receptor, TIGIT, inhibits the cytotoxic activity of natural killer (NK) cells against cancer cells, thus, allowing proliferation of the latter eventually leading to tumor growth. The aim of the study was to identify the immunogenicity of a peptide mimotope of the Fap2 protein and to determine the reactivity of colorectal cancer patients' sera against the mimotope.

Methods: Immunogenic epitope of the Fap2 protein of F. nucleatum was selected using the B-cell epitope prediction of the Immune Epitope Database and Analysis Resource (IEDB). The immunogenicity of the synthetic peptide mimotope of the Fap2 protein was determined in animal models and reactivity of colorectal cancer patients' sera against the mimotope was done by indirect ELISA.

Results: Results show that the selected peptide mimotope, with sequence TELAYKHYFGT, of the outer membrane protein Fap2 of $F$. nucleatum is immunogenic. Increase in the absorbance readings of peptide-immunized rabbit sera was observed starting Week 1 which was sustained up to Week 10 in the indirect ELISA performed. Colorectal cancer cases $(n=37)$ were all reactive in an ELISA-based analysis using the mimotope as the capture antigen.
\end{abstract}

Conclusions: In this study, we identified an immunogenic epitope of the Fap2 protein of the Fusobacterium nucleatum. We demonstrated the reactivity of serum of histopathologically confirmed CRC patients in a peptide-capture indirect ELISA which may serve as proof of concept for the development of CRC diagnostics.

Keywords: Fap2 protein, Fusobacterium nucleatum, ELISA, Colorectal cancer, Immunodiagnostics

\section{Background}

The composition of the gut microbiome has recently been implicated in the development of colorectal cancer (CRC) [1]. Dysbiosis, a condition characterized by a pathological imbalance in the microbial community, is shown to contribute in the growth and progression of colorectal tumors [2]. Several studies reported the high association of microorganisms belonging to the proteobacteria,

\footnotetext{
*Correspondence: laguevarra@ust.edu.ph

${ }^{1}$ Department of Biochemistry, Faculty of Pharmacy, University of Santo Tomas, Manila, Philippines

${ }^{2}$ Research Center for Natural and Applied Sciences, University of Santo

Tomas, Manila, Philippines

Full list of author information is available at the end of the article
}

such as Pseudomonas, Helicobacter, and Acinetobacter, with colon cancer [3]. The most current is that of Fusobacterium nucleatum, whose role in CRC progression has recently been described [4].

F. nucleatum is a non-spore forming, non-motile, gram-negative, spindle-shaped opportunistic anaerobic bacteria that can be found in the oral cavity and the gastrointestinal tract [5]. Its cell envelope is composed of outer and inner membranes flanking a periplasmic space and outer membrane proteins which comprise a third of its mass [6]. Originally identified as an oral commensal and a periodontal pathogen, F. nucleatum has recently been associated with several human illnesses 
which include adverse pregnancy outcomes, cardiovascular disease, rheumatoid arthritis, respiratory tract infections, Lemierre's syndrome, Alzheimer's disease, and gastrointestinal disorders including CRC [7]. The association of $F$. nucleatum with CRC development is attributed to the ability of patients' infected cancer cells to inhibit the ability of the immune system to attack tumoral cells $[7,8]$.

Fap2 protein is a 390-kilodalton protein encoded by the Fap2 gene of F. nucleatum [9]. It is an outer membrane protein composed of 3692 amino acid which is identified to induce apoptosis in human lymphocytes $[9,10]$. Recently, Fap2 protein has been shown to be involved in the binding of $F$. nucleatum to cancer cells and to interact with the immunoglobulin and ITIM domain (TIGIT) receptor mainly expressed on NK, Treg, CD8+ and CD4+ T cells [11]. The binding of Fap2 to TIGIT was found to inhibit the activity of natural killer (NK) cells against the tumor cells, thus causing the growth and progression of CRC [12].

Given this role of the F. nucleatum Fap2 protein in CRC tumorigenesis and the lack of a reported Fap2 immunogenic epitope in the Immune Epitope Database (IEDB) Resource Analysis (www.iedb.org), this study, therefore, sought to search for an immunogenic epitope of the $F$. nucleatum Fap2 protein to test the immunogenicity of a peptide mimotope in vivo and to measure the reactivity of plasma samples from confirmed CRC patients and clinically healthy controls to the mimotope.

\section{Methods}

Immune epitope prediction and peptide mimotope synthesis Prediction of the immune epitope of Fusobacterium nucleatum Fap2 protein was done in silico using the Immune Epitope Database (IEDB) Analysis Resource. The primary sequence of the Fap2 protein was taken from the National Center for Biotechnology Information (NCBI) Protein Database. The amino acid sequence of the epitope was selected using B-cell epitope prediction platform of the IEDB. Candidate immunogenic epitope was selected based on its antigenicity, surface accessibility, and hydrophilicity using Kolaskar \& Tongaonkar Antigenicity Scale and Emini Surface Accessibility Scale. The predicted immunogenic mimotope was synthesized by Genscript (New York, USA).

\section{Immunization}

Immunogen was prepared by conjugating the peptide mimotope to bovine serum albumin (BSA) following the methods of Coligan et al. [13]. Peptide conjugation in BSA (10:1) was done in the presence of glutaraldehyde in borate buffer ( $\mathrm{pH}$ 10) [13]. The BSA-conjugated peptide solution was reconstituted with PBS to produce a $1 \mathrm{mg} / \mathrm{mL}$ stock solution, which was then dialyzed against $4 \mathrm{l}$ of water for $24 \mathrm{~h}$.

Immunogenicity of the peptides was tested in 10- to 12-week-old white male New Zealand rabbits. Animal care and immunization were done following the protocols described by Bio-Synthesis (http://www.biosyn.com/) and as approved by the Institutional Animal Care and Utilization Committee (IACUC) of the University of Santo Tomas, Philippines. Six rabbits were randomly assigned to negative control $(n=2)$ and experimental $(n=$ 4) groups. Each rabbit from the experimental group was immunized with $1.0 \mathrm{~mL}$ solution containing $100 \mu \mathrm{g}$ of peptide distributed to five subcutaneous sites and two intramuscular sites. The immunogen was prepared by reconstituting the previously prepared peptide-BSA stock solution with PBS emulsified with Complete Freund's Adjuvant (Sigma). Four booster doses of the same amount of peptide emulsified with Incomplete Freund's Adjuvant (Sigma) were given at two weeks interval. Rabbits in the negative controls were given the same treatment except that the solutions used in the immunization did not contain the peptide mimotope.

\section{Immunogenicity assay}

Immunogenicity of the synthetic peptide mimotope of the Fap2 predicted epitope was analyzed by detecting presence of anti-peptide mimotope antibody in the sera of rabbits. Blood was collected through the marginal ear vein before immunization to serve as baseline, and after immunization at weekly intervals for 10 weeks for the immunogenicity assay. Serum was separated from the cells by centrifugation of the collected blood at $3400 \times$ g. Collected serum was transferred to Eppendorf tubes and stored at $-20{ }^{\circ} \mathrm{C}$ until use.

Presence of anti-peptide mimotope antibody was detected by indirect ELISA. Wells were coated with $50 \mu \mathrm{L}$ of coating buffer ( $0.05 \mathrm{M}$ carbonate buffer, $\mathrm{pH}$ 9.6) containing $10 \mu \mathrm{g}$ of peptides and blocked with $100 \mu \mathrm{L}$ of blocking solution ( $2 \%$ gelatin in $0.01 \mathrm{M}$ PBS containing $0.05 \%$ Tween 20). Fifty microliters of 1:100 diluted rabbit sera were then added to each well after washing with wash buffer (0.01 M PBS containing 0.05\% Tween 20) and incubated for $1 \mathrm{~h}$ at $37{ }^{\circ} \mathrm{C}$. The wells were washed again to remove the unbound rabbit antibodies. HRPconjugated goat anti-rabbit antibody (Genscript, New York) was used to detect presence of anti-peptide mimotope rabbit antibody using TMB as substrate.

\section{Reactivity assay}

Reactivity of sera collected from confirmed CRC cases $(n=37)$ and clinically healthy case-matched controls was also determined by indirect ELISA as previously mentioned. Patients' sera were also diluted 1:100 and the antipeptide mimotope human antibody was detected using HRP-conjugated anti-human antibody (Koma Biotech, Seoul, Korea). Protocols on the use of left-over plasma samples were approved by the Ethics Review Board of the University of Santo Tomas Hospital. 


\section{Statistical treatment and analysis}

Comparison of the mean absorbance readings from the indirect ELISA of negative control and experimental groups from baseline up to 10 weeks after the initial immunization was done by multivariate analysis using IBM SPSS Statistics version 20. $P$-values less than 0.05 were considered statistically significant.

The ELISA cut-off values were computed based on the formula described by Frey et al. [14]. Values higher than the computed cut-off were assessed as reactive while those that were lower were evaluated as non-reactive.

\section{Results}

In this study, selection of a candidate immune epitope was done using in silico analysis tools embedded in the Immune Epitope Database and Analysis Resource (IEDB). The sequence of the predicted immunogenic epitope was TELAYKHYFGT which is located at the 3596th to 3606th position of the Fap2 protein. Antigenicity and surface accessibility scores were 1.028 and 1.198, respectively, which were above the cut-off values 0.998 and 1.000 , respectively. The synthetic peptide mimotope of the selected immunogenic epitope was also reported to be soluble in

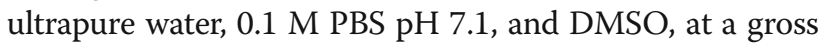
peptide concentration of less than $15 \mathrm{mg} / \mathrm{mL}$.

Immunogenicity of the synthetic peptide mimotope was evaluated by monitoring the weekly anti-peptide antibody titer in the animal models immunized with the peptide. Figure 1 presents the weekly mean absorbance readings of the negative control and peptide immunized groups.

The mean absorbance of the baseline blood samples of the peptide immunized and negative control groups were 0 . 052 and 0.055 , respectively. These readings were below the computed cut-off value of 0.072 . An increase in the absorbance was observed in the peptide-immunized group but not in the negative control group starting a week after the first immunization. A constant increase in anti-Fap2 titer was observed from Week 1 to Week 10 of immunization of the experimental rabbits. Independent $t$-test showed no significant difference in the absorbance readings of baseline blood samples collected from the normal control and peptide immunization group $(p$-value $=0.387$ ) However, multivariate analysis revealed statistically significant increase in the absorbance readings starting Week 1 after immunization as compared to the negative control $(p$-values $<0.02)$.

After confirming the immunogenicity of the synthetic peptide, we tested for the reactivity of plasma samples from histologically confirmed colorectal cancer cases and their age- and sex- matched clinically healthy controls to the peptide by indirect ELISA. Figure 2 presents the $\mathrm{OD}_{540}$ readings of CRC positive sera and their case-matched controls in contrast to the cut-off value of 0.05284 .

All cases $(n=37 ; 100 \%)$ showed reactivity to the Fap2 peptide mimotope. Twelve of the controls (32\%) also tested positive for IgG against the peptide (Table 1). These results gave a sensitivity of $100 \%$ and a specificity of $68 \%$. The positive predictive value and the negative predictive value are $76 \%$ and $100 \%$, respectively.

\section{Discussion}

In silico analysis tools provide a valuable platform in predicting and selecting candidate peptide sequences that can be used for immunodiagnostics and immunotherapeutics [15]. They have improved and greatly accelerated epitope design, which is a crucial step in vaccine as well as in diagnostic development [16]. When existing databases provide limited information on desired immunogenic epitopes, such as the case of the Fap2 protein of $F$. nucleatum, several immune epitope prediction tools, which are based on the physico-chemical properties of a portion of a polypeptide chain, can be used [17]. These physico-chemical properties include antigenicity,

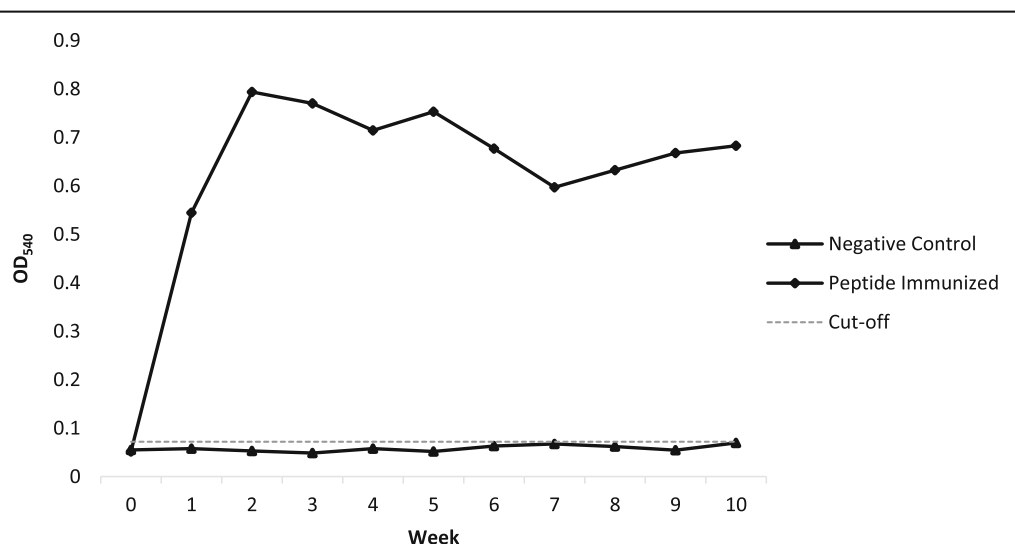

Fig. 1 Immunogenicity of a Fap2 Peptide Mimotope. Indirect ELISA absorbance readings of peptide immunized group as compared to the negative control and computed cut-off value 


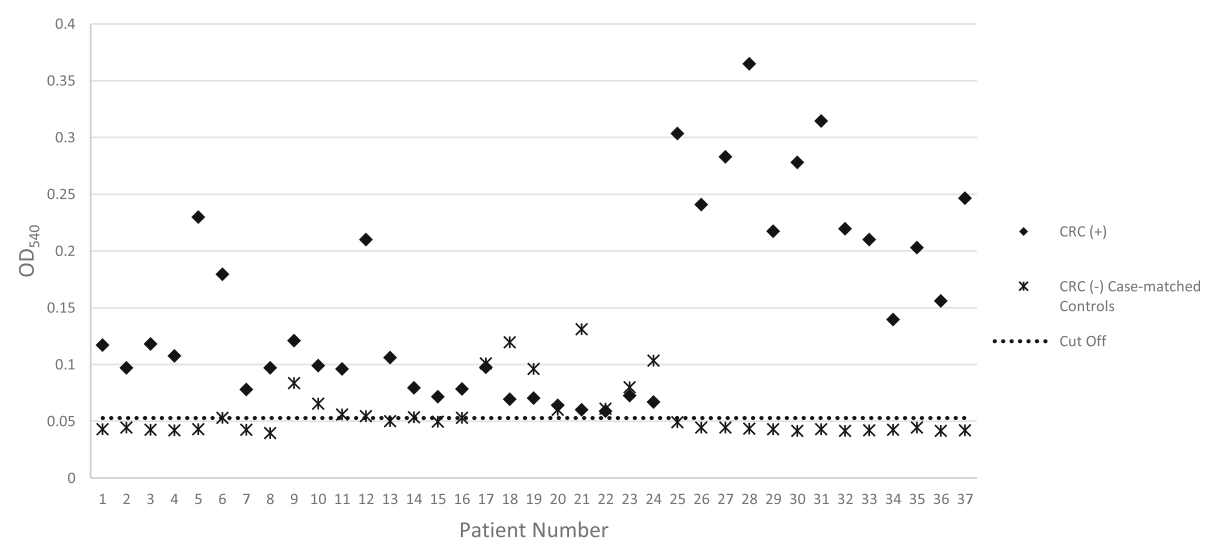

Fig. $2 \mathrm{OD}_{540}$ Readings of Colorectal Cancer Patients and Case-matched Healthy Controls. $\mathrm{OD}_{540}$ values higher than the computed cut-off value of 0.05284 were assessed as reactive. All CRC patients were reactive while 25 of the 37 case-matched controls have values lower than the cutoff

surface accessibility, and hydrophilicity based on the frequency of existing amino acids in the primary sequence of a target antigen [17-19].

In this study, we have shown in vivo the immunogenicity of the in silico-predicted immunogenic epitope of Fap2 antigen. The statistically significant increase in the absorbance readings in the ELISA of the mimotopeimmunized rabbits, a pattern which was not observed in negative control group, is indicative of the production of an anti-peptide IgG.

Peptides have the ability to induce an immune response and prompt the cell to produce antibodies [20]. Synthetic peptides can excellently mimic proteins because they are exact copies of protein fragments which are responsible for the protein's activity [21]. Synthetic peptide immunogens can induce an immune response by mimicking the activity of the subunit of the whole protein antigen and be used as a promising tool in vaccine and immunodiagnostic tool development [22]. Because of the simplicity, ease of use, and target specificity of utilizing peptide mimotopes, this procedure is preferred over other existing methods [22, 23]. Although one major challenge in using peptides as immunogen is its weak immunogenic property, this can be solved by conjugating it with carrier proteins such as BSA and keyhole limpet hemocyanin [24].

Table 1 Seroreactivity to Fusobacterium nucleatum Fap2 Peptide Mimotope of Colorectal Cancer Cases and Clinically Healthy Controls

\begin{tabular}{|c|c|c|c|c|c|c|}
\hline \multirow{2}{*}{$\begin{array}{l}\text { Seroreactivity to Fap2 } \\
\text { Peptide Mimotope }\end{array}$} & \multicolumn{2}{|c|}{ CRC Cases } & \multicolumn{2}{|c|}{ Healthy Controls } & \multicolumn{2}{|c|}{ Total } \\
\hline & $\bar{n}$ & $\%$ & $\bar{n}$ & $\%$ & $\bar{n}$ & $\%$ \\
\hline Reactive & 37 & $100 \%$ & 12 & 32 & 49 & $66 \%$ \\
\hline Non-reactive & 0 & 0 & 25 & 68 & 25 & $34 \%$ \\
\hline Total & 37 & $100 \%$ & 37 & $100 \%$ & 74 & $100 \%$ \\
\hline
\end{tabular}

Synthetic peptides mimicking immunogenic epitopes of protein antigens can initiate and activate the immune response by binding to either the Class I or Class II major histocompatibility complex (MHC) [25]. In 2005, Wang et al. demonstrated the ability of peptide mimotopes prepared from MHC Class I library of baculovirus to bind to T-cell and secret interleukin-2 [26]. In the same year, Gevorkian et al. were able to elicit polyclonal antibodies against a cell surface antigen of Mycobacterium tuberculosis in rabbits using a 15-mer chemically synthesized peptide [27]. Buchwald et al (2005), in their search for a potential vaccine against pneumococcus, observed that a 12-mer peptide elicited production of antibodies in mice models which were then seroprotected during the infection challenge [28]. The anti-tumor activity of peptide mimotopes through the production of anti-HBJ127, a tumor suppressing antiCD98 heavy chain antibody, was observed by Saito et al. [29].

Synthetic peptides are also used in ELISA in the diagnosis of diseases [30]. Antibodies present in serum of immunized or previously infected individuals reacts to the peptides in immunoassays [31-33]. This was also observed in our experiments when we used the peptide as the capture antigen in the ELISA performed to detect presence of anti-peptide antibody in the sera of peptide-immunized rabbits as well as patients with colorectal carcinoma - a disease associated with the presence of harmful microbiota, such as $F$. nucleatum, in the gut.

The human gut is home to a complex system of microorganisms which play a vital role in the host's health homeostasis [2]. Recent studies suggest the role of the gut microbiota in the development in colorectal cancer due to their ability to interfere in the host's inflammatory and immunomodulatory activities, which subsequently favor carcinogenesis and tumorigenesis [33-36]. Among the recently studied and implicated in colorectal carcinoma 
development is F. nucleatum [37, 38]. This bacterium has also been associated with chemoresistance of cancer cells during treatment [38]. Hence, there is the need to develop methods for their detection as well as determine the anti- $F$. nucleatum titer that is associated with CRC.

F. nucleatum's outer membrane protein Fap2 is identified to adhere to host's cell surface and induce proinflammatory and pro-carcinogenic response [39]. Its association to the development of colorectal cancer and its role in immune evasion was first reported by Kaplan et al. [40] when they observed that $F$. nucleatum proteins induce lymphocyte apoptosis. This was further supported by Mima et al. [8] when they observed that the number of $F$. nucleatum cells in CRC cases was observed to be inversely associated with the density of $\mathrm{CD}^{+} \mathrm{T}$-cells. Recently, proposed roles and molecular mechanisms of $F$. nucleatum outer membrane proteins in immune evasion have been reported. The ability of $F$. nucleatum to bind colorectal adenocarcinoma cells was reported previously [41]. It was also observed that the binding of Fap2 to human inhibitory receptor TIGIT protects the cancer cells from the immunosurveillance activity of NK cells, which, in effect, would allow the cancer cells to grow and proliferate [11]. These studies serve as impetus in understanding the role and mechanism of Fap2 protein in the colorectal carcinogenesis.

The ability to identify the immunogenic epitopes of the Fap2 of F. nucleatum is relevant in developing immunodiagnostic and therapeutic methods for CRC. The immunogenicity of and the reactivity of the CRC patients' plasma samples to the peptide mimotope can serve as starting point in developing methods in the screening and detection CRC.

\section{Conclusions}

We have identified an immunogenic epitope of the Fap2 protein of $F$. nucleatum. The peptide TELAYKHYFGT located at the 3596th to 3606th amino acid of the outer membrane protein Fap2 of the CRC-associated pathogen $F$. nucleatum proved to be immunogenic in animal models. Seroreactivity of CRC patients against the peptide mimotope in an indirect ELISA was also observed.

Identifying immunogenic epitopes of CRC-associated pathogens such as $F$. nucleatum may serve as impetus in the development of vaccine and immunodiagnostic tools against colorectal cancer. Future studies may use results from this study to pursue a pathogen-targeted control of cancer similar to the human papilloma virus (HPV) or as a biomarker for detection and determination of risks of cancer development similar to Eppstein Barr virus.

\section{Abbreviations}

BSA: bovine serum albumin; CRC: Colorectal cancer; ELISA: Enzyme-linked immunosorbent assay; HRP: horseradish peroxidase; IACUC: Institutional Animal Care and Utilization Committee; IEDB: Immune epitope database; IgG: Immunoglobulin G; NCBI: National Center for Biotechnology Information; NK: Natural killer; PBS: phosphate buffered saline; TIGIT: T cell immunoglobulin and ITIM domain (TIGIT); TMB: 3,3',5,5'-Tetramethylbenzidine

\section{Acknowledgments}

The authors would like to acknowledge the support of the Department of Biochemistry, Faculty of Pharmacy, University of Santo Tomas and the Research Center for Natural and Applied Sciences for allowing them to use their facilities.

\section{Funding}

This research was done as an independent individual initiative of the authors. No funding was received from any research grant institution from the Philippines or abroad except for the in-house financial assistance provided by the Research Center for Natural and Applied Sciences (RCNAS) of the University of Santo Tomas to RCNAS-affiliated researchers.

\section{Availability of data and materials}

All data collected and analyzed in this study will be made available to the reviewers by the corresponding author upon request.

\section{Authors' contributions}

The following are the contributions of the individual authors: LAG. Corresponding author, primarily responsible for the preparation of the manuscript, design of the protocols used in the experiment and analysis of results. ACFA. Data collection and preliminary analysis of results. Primarily responsible in peptide selection. PJOB. Data collection and preliminary analysis of results, primarily responsible for animal care and utilization. Also involved in troubleshooting during optimization of ELISA protocols. KJSD. Data collection and preliminary analysis of results, primarily responsible in writing the preliminary manuscript. SJQL. Data collection and preliminary analysis of results, primarily responsible in performance of ELISA and troubleshooting during optimization.TTS. Data collection and review of the manuscript. PMSPA. PhD.Conceptualization, troubleshooting of the immunobased assay, and internal review of the paper. All authors read and approved the final manuscript.

\section{Author's information}

Mr. Leonardo A. Guevarra Jr., the principal investigator of this project and the corresponding author for this study, is a faculty member at the Department of Biochemistry, Faculty of Pharmacy and Research Faculty at the Research Center for Natural and Applied Sciences, University of Santo Tomas. He first got his interest in infectious disease, cancer, and diagnostics development when he was doing his research for the Master of Science in Biochemistry at the Department of Biochemistry and Molecular Biology, College of Medicine, University of the Philippines - Manila. His current organizational involvement includes the Philippine Society of Biochemistry and Molecular Biology (lifetime member), UK Biochemical Society, and the Japanese Peptide Society where he recently presented (poster presentation) the results in the paper being submitted for publication. Mr. Guevarra is establishing his research career in immune epitope prediction and its application in infectious disease and cancer diagnostics.

\section{Ethics approval and consent to participate}

This study was approved by the Institutional Review Board of the University of Santo Tomas Hospital chaired by Dr. Wilson L. Tan De Guzman. You can contact the IRB by email at ust_irb@yahoo.com.ph. Written informed consent for the use of leftover serum collected from routine serological testing was requested from patients participating in the study. Clearance and approval from the Institutional Animal Care and Utilization Committee of the University of Santo Tomas was acquired. The UST IACUC is chaired by Prof. Jovencio G. Apostol, PhD and can be contacted through ustrcns@yahoo.com.

Consent for publication

Not applicable. 


\section{Competing interests}

The authors declare that they have no competing interests.

\section{Publisher's Note}

Springer Nature remains neutral with regard to jurisdictional claims in published maps and institutional affiliations.

\section{Author details}

${ }^{1}$ Department of Biochemistry, Faculty of Pharmacy, University of Santo Tomas, Manila, Philippines. ${ }^{2}$ Research Center for Natural and Applied Sciences, University of Santo Tomas, Manila, Philippines. ${ }^{3}$ Benavidez Cancer Institute, University of Santo Tomas Hospital, Manila, Philippines. ${ }^{4}$ Department of Biological Sciences, College of Science, University of Santo Tomas, Manila, Philippines.

\section{Received: 23 January 2018 Accepted: 21 March 2018} Published online: 02 April 2018

\section{References}

1. Lucas C, Barnich N, Nguyen HTT. Microbiota, inflammation and colorectal cancer. Int J Mol Sci. 2017;18(6). https://doi.org/10.3390/ijms18061310.

2. Dulal SK, Keku TO. Gut microbiome and colorectal adenomas. Cancer J. 2015:20(3):225-31.

3. Sanapareddy N, Legge RM, Jovov B, McCoy A, Burcal L, Araujo-Perez F, Keku TO. Increased rectal microbial richness is associated with the presence of colorectal adenomas in humans. ISME J. 2012;6(10):1858-68.

4. Gholizadeh P, Eslami H, Kafil HS. Carcinogenesis mechanisms of Fusobacterium nucleatum. Biomed Pharmacother. 2017;89:918-25.

5. Bakken V, Høgh BT, Jensen HB. Growth conditions and outer membrane proteins of Fusobacterium nucleatum. Scand J Dent Res. 1990:98:215-24.

6. Bolstad Al, Jensen HB, Bakken V. Taxonomy, biology, and periodontal aspects of Fusobacterium nucleatum. Clin Microbiol Rev. 1996:9(1):55-71.

7. Han YW. Fusobacterium nucleatum: a commensal-turned pathogen. Curr Opin Microbiol. 2015:23:141-7.

8. Mima K, Sukawa Y, Nishihara R, Qian ZR, Yamauchi M, Inamura K, Ogino S. Fusobacterium nucleatum and T cells in colorectal carcinoma. JAMA Oncol. 2015;1(5):653.

9. Coppenhagen-Glazer S, Sol A, Abed J, Naor R, Zhang X, Han YW, Bachrach G. Fap2 of fusobacterium nucleatum is a galactose-inhibitable adhesin involved in coaggregation, cell adhesion, and preterm birth. Infect Immun. 2015:83:1104-13. [PubMed: 25561710]

10. Nosho K, Sukawa Y, Adachi $Y$, Ito M, Mitsuhashi K, Kurihara H, et al. Association of Fusobacterium nucleatum with immunity and molecular alterations in colorectal cancer. World J Gastroenterol. 2016;22(2):557-66.

11. Kaplan CW, Ma X, Paranjpe A, Jewett A, Lux R, Kinder-Haake S, Shi W. Fusobacterium nucleatum outer membrane proteins Fap2 and RadD induce cell death in human lymphocytes. Infect Immun. 2010;78(11):4773-8.

12. Gur C, Ibrahim Y, Isaacson B, Yamin R, Abed J, Gamliel M, et al. Binding of the Fap2 protein of Fusobacterium nucleatum to human inhibitory receptor TIGIT protects tumors from immune cell attack. Immunity. 2015;42(2):344-55.

13. Coligan JE. Current Protocols in Immunology. New York: Wiley; 2001.

14. Frey A, Di Canzio J, Zurakowski D. A statistically defined endpoint titer determination method for immunoassays. J Immunol Methods. 1998:221(1-2):35-41.

15. Fleri W, Paul S, Dhanda SK, Mahajan S, Xu X, Peters B, Sette A. The immune epitope database and analysis resource in epitope discovery and synthetic vaccine design. Front Immunol. 2017;8(MAR):1-16. https://doi.org/10.3389/ fimmu.2017.00278.

16. Flower DR. Designing immunogenic peptides. Nat Chem Biol. 2013;9(12): 749-53.

17. Emini EA, Hughes JV, Perlow DS, Boger J. Induction of hepatitis a virusneutralizing antibody by a virus-specific synthetic peptide. J Virol. 1985;55(3): 836-9.

18. Kolaskar AS, Tongaonkar PC. A semi-empirical method for prediction of antigenic detetermininants on protein antigens. Febbs Lett. 1990;276(1,2): 172-4.

19. Parker JMR, Guo D, Hodges RS. New hydrophilicity scale derived from highperformance liquid chromatography peptide retention data: correlation of predicted surface residues with antigenicity and X-ray-derived accessible sites. Biochemist. 1986;25(19):5425-32.
20. Wallach J. Peptide antigen: a practical approach. In: Wisdom GB, editor. Biochemistry and molecular biology education. Oxford: Oxford University Press; 1996. p. 244-52.

21. Groß A, Hashimoto C, Sticht H, Eichler J. Synthetic peptides as protein mimics. Front Bioeng Biotechnol. 2016;3:211. https://doi.org/10.3389/fbioe. 2015.00211.

22. Meloen RH, Puyk WC, Meijer DJA, Lankhof H, Posthumus WPA, Antigenicity SWMM. Immunogenicity of synthetic peptides of foot and mouth disease. J Gen Virol. 1987:68:305-14.

23. Van Regenmortel MHV. Antigenicity and immunogenicity of synthetic peptides. Biologicals. 2001;29(3-4):209-13.

24. Azmi F, Fuaad AAHA, Skwarczynski M, Toth I. Recent progress in adjuvant discovery for peptide-based subunit vaccines. Hum Vaccin Immunother. 2014;10(3):778-96.

25. Blum J, Wearsch P, Cresswell P. Pathways of antigen processing. Annu Rev Immunol. 2013;31(1):443-73.

26. Wang $Y$, Rubtsov $A$, Heiser $R$, White J, et al. Using a baculovirus display library to identify MHC class I mimotopes. PNAS. 2005;102(7):2476-81.

27. Gevorkian G, Segura E, Acero G, Palma JP, et al. Peptide mimotopes of mycobacterium tuberculosis carbohydrate immunodeterminants. Biochem J. 2005;387(2):411-7.

28. Buchwald UK, Lees A, Steinitz M, Pirofski L. A peptide mimotope of type 8 pneumococcal capsular polysaccharide induces a protective immune response in mice. Infect Immun. 2005;73(1):325-33.

29. Saito $M$, Kondo M, Ohshima M, Deguchi K, et al. Identification of anti-CD98 antibody mimotopes for inducing antibodies with antitumor activity by mimotope immunization. Cancer Sci. 2014;105(4):396-401.

30. Ma F, Zhang L, Wang Y, Lu R, et al. Development of a peptide ELISA for the diagnosis of aleutian mink disease. PLoS ONE. 2016;11(11):e0165793. https:// doi.org/10.1371/journal.pone.0165793.

31. Sharma A, Saha A, Bhattacharjee S, Majumdar S, Das Gupta SK. Specific and randomly derived immunoactive peptide mimotopes of mycobacterial antigens. Clin Vaccine Immunol. 2006:13(10):143-1154

32. Chen J, Domingue JC, and Sears CL. Microbiota dysbiosis in select human cancers: evidence of association and causality. Semin Immunol. 2017; (July), 0-1. https://doi.org/10.1016/jsmim201708.001

33. Kang M, Martin A. Microbiome and colorectal cancer: Unraveling hostmicrobiota interactions in colitis-associated colorectal cancer development. Semin Immunol. 2017;(February):0-1. https://doi.org/10.1016/jsmim201704.003.

34. Fardini Y, Wang X, Temoin S, Nithianantham S, Lee D, Shoham M, Han WY. Fusobacterium nucleatum adhesin FadA binds vascular- endothelial cadherin and alters endothelial integrity. Mol Microbiol. 2012;82(6):1468-80.

35. Van Raay T, Allen-Vercoe E. Microbial Interactions and Interventions in Colorectal Cancer. Microbiol Spect. 2017:5:3. https://doi.org/10.1128/ microbiolspec.BAD-0004-2016. Review

36. Yoon H, Kim N, Park JH, Kim YS, Lee J, Kim HW, et al. Comparisons of gut microbiota among healthy control, patients with conventional adenoma, sessile serrated adenoma, and colorectal Cancer. J Cancer Prev. 2017:22(2):108-14.

37. Zeller G, Tap J, Voigt AY, Sunagawa S, Kultima JR, Costea PI, et al. Potential of fecal microbiota for early-stage detection of colorectal cancer. Mol Syst Biol. 2014;10(11):766

38. Yu T, Guo F, Yu Y, Sun T, Ma D, Han J, et al. Fusobacterium nucleatum promotes chemoresistance to colorectal cancer by modulating autophagy. Cell. 2017:170(3):548-63.e16

39. Rubinstein MR, Wang X, Liu W, Hao Y, Cai G, Wan Y. Fusobacterium nucleatum promotes colorectal carcinogenesis by modulating E-cadgerin/ $\beta$ catein signalling via its FadA adhesin. Cell Host Microbe. 2013:14(2):195-206.

40. Kaplan CW, Lux R, Huynh T, Jewett A, Shi W, Haake SK. Fusobacterium nucleatum apoptosis-inducing outer membrane protein. J Dent Res. 2005; 84(8):700-4

41. Abed J, Emgård JEM, Zamir G, Faroja M, Grenov A, Sol A, et al. Fap2 mediates Fusobacterium nucleatum colorectal adenocarcinoma enrichment by binding to tumor-expressed gal-GalNAc. Cell Host Microbe. 2017;20(2): 215-25. 